\title{
HOSPITALIZAÇÕES POR NEOPLASIAS EM IDOSOS NO ÂMBITO DO SISTEMA ÚNICO DE SAÚDE NA PARAÍBA/BRASIL
}

Rilva Lopes de Sousa-Muñoz

Docente associado na Universidade Federal da Paraíba (UFPB); Doutora em Produtos Naturais e Sintéticos Bioativos Universidade Federal da Paraíba (UFPB), Brasil.

E-mail: rilvalopes@hotmail.com

\section{Maria Yvone Queiroz Formiga \\ Ana Elisa Vieira Fernandes Silva \\ Mirella Bezerra de Lima Silva \\ Raiara Carvalho Vieira \\ Miéllio Melo Galdino}

Discentes de Medicina pela Universidade Federal da Paraíba (UFPB), Brasil.

\section{Melissa Toscano Montenegro de Morais}

Discente do curso de Medicina na Universidade Federal da Paraíba (UFPB), Brasil; Bolsitas de Iniciação Científica do Programa Jovens Talentos para a Ciência do CNPQ, Brasil.
RESUMO: O objetivo deste estudo foi avaliar a frequência de hospitalizações de pacientes idosos com diagnóstico principal de câncer no sistema público de saúde do Estado da Paraíba, Brasil, entre 2008 e 2013, e verificar as localizações mais frequentes da doença e sua mortalidade hospitalar. Realizou-se estudo descritivo e retrospectivo, com análise das internações de idosos que tiveram câncer como causa de hospitalização no período entre janeiro de 2008 e janeiro de 2013 no Sistema Único de Saúde (SUS). Os registros foram obtidos na plataforma Datasus-Net, do Ministério da Saúde, a partir do Sistema de Informações Hospitalares (SIH) e do Sistema de Informação sobre Mortalidade (SIM). O número de casos foi de 10.739, o que representa $25,4 \%$ das hospitalizações por câncer em todas as faixas etárias. A frequência de diagnósticos de neoplasias foi de 4,5\% em relação ao número total de internações de idosos no mesmo período, considerando todas as categorias da CID-10. Entre as hospitalizações por câncer em idosos, 51,7\% foram do sexo masculino. A maioria dos casos foi observada entre 60 e 69 anos. As localizações mais frequentes foram próstata, mama e cólon. Ocorreram 1.448 óbitos (13,5\% dos casos), com maior taxa de mortalidade por câncer de próstata. Os resultados encontrados indicam prevalência das internações hospitalares na população idosa na Paraíba por câncer inferior aos de outros Estados do Brasil, mas representando percentual importante em relação ao número total de hospitalizações por câncer no Estado, com maiores frequências de câncer de próstata, mama e cólon, com elevada mortalidade hospitalar.

PALAVRAS-CHAVE: Câncer; Epidemiologia; Hospitalização; Saúde do Idoso.

\section{ELDERLY PEOPLE'S HOSPITALIZATION DUE TO CANCER IN THE NATIONAL HEALTH SERVICE IN PARAÍBA, BRAZIL}

\begin{abstract}
Current analysis evaluates the frequency of hospitalizations of the elderly due to cancer, in the National Health Service in the state of Paraíba, Brazil, between 2008 and 2013, and verifies the disease's loci and mortality in hospital. A descriptive and retrospective analysis was conducted on the hospitalization of elderly people with cancer as the main cause for their hospitalization between January 2008 and January 2013 by SUS. Registers were obtained from Datasus-Net of the Ministry of Health derived from the System of Hospital Information (SIH) and Information System on Mortality (SIM). The number of cases amounted to 10,739 , or rather, $25.4 \%$ of hospitalization for cancer in all age brackets. Cancer diagnose frequency was $4.5 \%$ out of the total number of hospitalization of elderly people during the same period, for all CID-10 categories. Hospitalizations of elderly males for cancer
\end{abstract}


reached $51.7 \%$, with most cases occurring between 60 and 69 years old. Most frequent sites were prostate, breast and cervical colon. Results showed 1,448 deaths (13.5\% of cancer cases), with highest mortality rate for prostate cancer. Results revealed a lower prevalence of hospital hospitalizations in the elderly people of Paraíba by cancer when compared to that in other Brazilian states. However, they make up a significant percentage with regard to total number of hospitalizations for cancer in the state, with a higher frequency in prostate, breast and colon cancer, with high mortality rates.

KEY WORDS: Epidemiology; Cancer; Health of the Elderly; Hospitalization.

\section{INTRODUÇÃO}

$\mathrm{O}$ rápido processo de envelhecimento da população brasileira constitui um relevante desafio para as políticas públicas de saúde atualmente. Frente a esta nova realidade demográfica, destaca-se a necessidade de estruturação de serviços e de programas de saúde para responder às demandas emergentes (LOYOLA FILHO et al., 2004). O risco de hospitalizações na população idosa é mais alto que o dos adultos mais jovens (20-59 anos), tanto no que se refere às taxas gerais quanto às taxas específicas por causa (LOYOLA FILHO et al.; SIQUEIRA et al., 2004).

Segundo Vallgarda (1999), as mudanças nos diagnósticos de pacientes idosos durante a última década ilustram modificações nos padrões de doenças e de hospitalizações, com aumento progressivo na incidência de câncer com o avanço da idade. Portanto, o envelhecimento da população provavelmente tem como implicação diferentes necessidades de cuidados de saúde também no que se refere à maior prevalência de neoplasias. Estima-se que em 2020, 70\% das neoplasias ocorrerão em indivíduos com idade superior a 60 anos (AMERICAN CANCER SOCIETY, 2005; ASSIS et al., 2011).

No Brasil, a distribuição dos diferentes tipos de neoplasias sugere uma transição epidemiológica em andamento. Esta frequência é variável em função da região considerada do país, o que enfatiza a necessidade do estudo das variações geográficas nos padrões desta doença. Contextualizando em nossa realidade, é importante salientar que a Paraíba é o terceiro Estado no Brasil com maior número relativo de idosos, e o segundo no Nordeste segundo dados do IBGE (2010), porém não há estudos publicados sobre a demanda da população desta faixa etária no Estado.

Frente ao exposto, e uma vez que o processo de envelhecimento tenderá a se aprofundar nas próximas décadas, torna-se importante a realização de estudos que abordem doenças crônicas não transmissíveis associadas à velhice, com dados atualizados referentes aos tipos de neoplasias que demandam internações hospitalares por pessoas idosas.

O objetivo do presente trabalho foi avaliar a frequência de hospitalizações de pacientes idosos com diagnóstico principal de câncer no âmbito do Sistema Único de Saúde (SUS), no Estado da Paraíba entre 2008 e 2013, além de verificar as localizações mais frequentes da doença e a sua mortalidade hospitalar em uma perspectiva temporal.

\section{METODOLOGIA}

\subsection{MODELO DO ESTUDO}

Estudo de modelo descritivo e retrospectivo, com análise de dados secundários em saúde através de análise das internações no SUS.

\subsection{AMOSTRAGEM}

Usuários do Sistema Único de Saúde (SUS) com 60 anos ou mais e cadastrados no sistema de internações hospitalares (SIH) no Estado da Paraíba foram selecionados para fazer parte do estudo, devido à maior susceptibilidade às neoplasias malignas nessa faixa etária. Foi realizado um levantamento do número de internações hospitalares e óbitos em idosos por diferentes tipos de neoplasmas (tumores), segundo a Classificação Internacional das Doenças - 10 (CID10), capítulo II, abrangendo os códigos de $\mathrm{C} 00$ a $\mathrm{C} 97$ (neoplasias malignas).

Segundo o Censo do IBGE em 2010, o Estado da Paraíba possuía uma população de 3.766 .528 habitantes, 
constituindo o quinto Estado mais populoso do Nordeste brasileiro. Esse contingente populacional correspondia a $1,97 \%$ da população nacional, e cujo percentual de idosos era de $12 \%$ (451.101) naquele Censo.

A amostragem deste estudo foi constituída pelos dados coletados na plataforma Datasus-Net, do Departamento de Informática do Sistema Único de Saúde (Datasus), sobre todas as internações hospitalares de idosos no Estado da Paraíba no período de cinco anos. As causas de internação no Sistema Único de Saúde (SUS) são importante fonte de informações para o planejamento e as decisões dos seus gestores. Neste estudo, buscou-se verificar a frequência de internações hospitalares associadas a câncer em idosos nos serviços públicos e conveniados de saúde na Paraíba, a partir de estatísticas hospitalares, como o Sistema de Informações sobre Autorização de Internações Hospitalares (SIH) e o Sistema de Informação sobre Mortalidade (SIM), produzidos pelo Ministério da Saúde (MS), que representam a fonte oficial do Ministério da Saúde para cálculo de indicadores que analisam as internações hospitalares, um sistema informatizado, contendo dados das hospitalizações de todos os pacientes.

Foram analisados os dados referentes às internações de pacientes de 60 anos ou mais, porém a unidade de análise foi a alta hospitalar (registros das Autorizações de Internações Hospitalares - AIH). Os critérios de inclusão foram todos os dados contidos no SIH/SUS, em AIHs de hospitalizações de idosos no Estado da Paraíba, Brasil, de pacientes com 60 anos de idade ou mais, e cuja hospitalização tivesse ocorrido no âmbito do SUS no Estado da Paraíba entre os anos de 2008 e 2013, período em que se consideraram os dados disponíveis. Foram consideradas idosas as pessoas com mais de 60 anos, de acordo com a legislação brasileira vigente (LIMA E COSTA et al., 2000).

As internações foram apresentadas por capítulo da Classificação Internacional de Doenças (CID-10) e conforme Lista de Tabulação para Morbidade oferecida no SIH/SUS, segundo consta nas normas técnicas disponibilizadas pelo sistema. Ressalta-se que a causa de internação é a informada como diagnóstico principal, definido como sendo o que motivou a hospitalização.

\subsection{PROCEDIMENTOS DE COLETA DE DADOS}

Os registros referentes às hospitalizações por neoplasias em idosos na Paraíba foram obtidos na plataforma Datasus-Net, a partir do Sistema de Informações Hospitalares (SIH) e do Sistema de Informação sobre Mortalidade (SIM) no Brasil, através de acesso às seguintes páginas, respectivamente:

- SIH: http:/tabnet.datasus.gov.br/cgi/ deftohtm.exe?sih/cnv/nipb.def

- SIM: http://tabnet.datasus.gov.br/cgi/ deftohtm.exe?idb2012/c10.def

OSIH fornece, a partirdaAIH, dados demográficos e clínicos, permitindo descrever a morbidade hospitalar e a mortalidade no âmbito dos serviços próprios e conveniados ao SUS. Portanto, não foram incluídas as internações da população usuária de planos de saúde privados. O SIM fornece dados a partir das declarações de óbito coletadas pelas Secretarias Estaduais de Saúde, através dos Indicadores e Dados Básicos de 2012.

Foram construídas planilhas que possibilitaram a descrição de dados, com padrões dos municípios, de acordo com os seguintes procedimentos:

1. Acessaram-se os arquivos do SIH/SUS, referentes à produção hospitalar do Estado da Paraíba no período de cinco anos, de janeiro de 2008 a janeiro de 2013, através do instrumento de tabulação TABWIN (aplicativo tabulador do Datasus), com a devida seleção dos dados pertinentes à pesquisa.

2. Realizaram-se os levantamentos, separadamente, por parâmetro: total de internações de idosos, hospitalizações por câncer em todas as idades e hospitalizações por câncer em idosos. Em relação a estas últimas, pesquisaram-se as frequências por sexo, localidade onde ocorreu a internação (João Pessoa, Campina Grande, outros municípios da Paraíba), capítulo da CID-10 (neoplasias [tumores]), localização do câncer (lista de morbidades da CID-10), regime (público ou 
privado conveniado), permanência hospitalar (médias), óbitos (total e por neoplasias) e caráter da internação (eletivo, urgência).

3. Organizou-se uma planilha contendo os dados de interesse (variáveis) em um programa do software Statistical Package os Social Sciences (SPSS) no formato de banco de dados, com preenchimento dos valores numéricos obtidos a partir do aplicativo do Datasus-Net, para facilitar a visualização e posterior análise comparativa das informações coletadas.

4. Acessaram-se os arquivos do SIM/SUS, referentes às taxas de mortalidade específica disponíveis até o dia 11 de junho de 2014.

Utilizaram-se os três blocos de faixa etária que agrupam os idosos na composição de intervalos de nove em nove anos de idade: dos 60 aos 69 anos; dos 70 aos 79 anos; e com 80 anos ou mais. O diagnóstico analisado foi aquele relatado como principal e registrado como neoplasia em cada AIH. Foi registrado também o número total de internações de idosos em cada ano para cálculo da taxa de prevalência de câncer como causa de internação nesta clientela, assim como o número total de casos registrados de internação por neoplasias em todas as idades, para verificar a proporção de neoplasias malignas em idosos em relação ao total de internações.

As taxas de internação não foram calculadas, uma vez que a cobertura do SUS não é completa, impossibilitando a identificação da população sob risco (denominador) para este cálculo. Desta forma, foram consideradas as internações proporcionais, ou seja, aquelas para as quais o denominador foi o total das internações de idosos na Paraíba no período considerado.

Foram coletadas as taxas de mortalidade específica (óbitos por 100.000 habitantes) por neoplasias a partir dos Indicadores de Mortalidade do SIM, onde estão disponíveis as taxas referentes a sete tipos de câncer (pulmão, esôfago, estômago, reto e ânus, mama feminina, colo de útero e próstata), além da taxa referente às neoplasias das demais localizações, para os anos de 2008, 2009, 2010 e 2011. A taxa de mortalidade específica por neoplasias do colo do útero e de mama foram calculadas apenas para a população feminina, e por neoplasias da próstata, apenas para a população masculina.

A coleta e a análise dos dados na plataforma Datasus-Net foram realizadas no período de julho de 2013 a julho de 2014.

\subsection{VARIÁVEIS}

As variáveis do estudo foram: (a) variável primária: prevalência de diagnóstico de câncer em pacientes idosos hospitalizados pelo SUS na Paraíba; (b) secundárias: sexo; faixas de idade (60-69; 70-79; 80 e mais); localização do câncer; regime (público, privado conveniado); local de internação; permanência hospitalar; e mortalidade hospitalar.

\subsection{ANÁLISE ESTATÍSTICA}

As informações colhidas a partir do SIH/SUS foram organizadas em um banco de dados utilizando o SPSS para Windows, versão 17.0, e sumarizados por análise descritiva, sendo utilizadas frequências absolutas e relativas para variáveis categóricas, e médias para variáveis contínuas, sendo dispostas em tabelas e representações gráficas. Realizou-se análise de correlação bivariada das frequências entre os sexos. Na estatística inferencial, realizou-se análise de variância para comparação dos números médios de internamentos de idosos e dos óbitos entre os anos de 2008 e 2013, adotando-se um nível de significância estatística de 5\%.

\subsection{CONSIDERAÇÕES ÉTICAS}

Trata-se de um estudo retrospectivo com compilação e análise de dados secundários, de domínio público, não havendo, portanto, necessidade de submissão do projeto a análise de comitê de ética em pesquisa.

\section{RESULTADOS}

A frequência de hospitalizações de pacientes idosos com diagnóstico principal de câncer no SUS no 
Estado da Paraíba (hospitais públicos e conveniados) entre $1^{\circ}$ de janeiro de 2008 e $1^{\circ}$ de janeiro de 2013 (cinco anos) foi de 10.739 , que representa $25,4 \%$ das hospitalizações por câncer em todas as faixas etárias no referido período $(\mathrm{n}=42.336)$. Por outro lado, a frequência percentual de diagnósticos de neoplasias representou $4,5 \%$ do número total de internações de idosos no mesmo período, considerando todas as categorias da CID-10 $(n=236.277)$.

A observação da curva representativa dos números de casos de internações associadas a neoplasias malignas por ano apresentou formato ascendente, porém sem diferenças estatísticas significantes entre os anos através da análise de variância $(p=0,469)$, como demonstra a Figura 1.

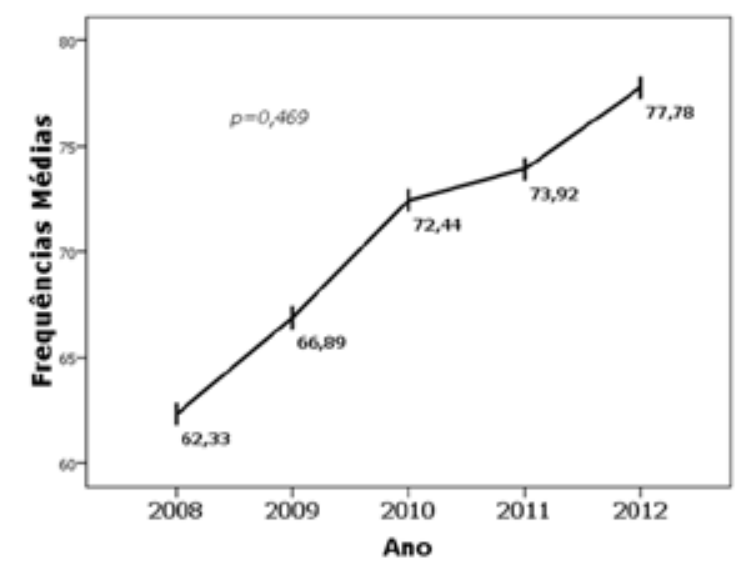

Figura 1. Frequências médias de internamentos por câncer entre os idosos atendidos no Sistema Único de Saúde (SUS) na Paraíba por ano entre 2008 e 2013 (mês de janeiro excluído).

Entre as hospitalizações por câncer em idosos, 6.698 (62,3\%) foram de pacientes do sexo masculino. A maioria dos casos foi observada na faixa de idade entre 60 e 69 anos, seguida daqueles com 70 a 79 , com menor frequência na faixa de 80 e mais (Tabela 1 ).
Tabela 1. Frequências de Hospitalizações com Diagnóstico Principal de Câncer entre os Idosos Atendidos no Sistema Único de Saúde (SUS) na Paraíba no Período entre Janeiro de 2008 e Janeiro de 2013

\begin{tabular}{lcc}
\hline & Faixas de Idade & \multicolumn{2}{c}{ Frequências } \\
& $\boldsymbol{f}$ & $\%$ \\
\hline $60-69$ & 5.446 & 50,7 \\
$70-79$ & 3.747 & 34,9 \\
80 e mais & 1.546 & 14,4 \\
Total & 10.739 & 100 \\
\hline
\end{tabular}

As frequências médias de casos no sexo masculino foram superiores às do feminino em todos os anos avaliados (Figura 2).

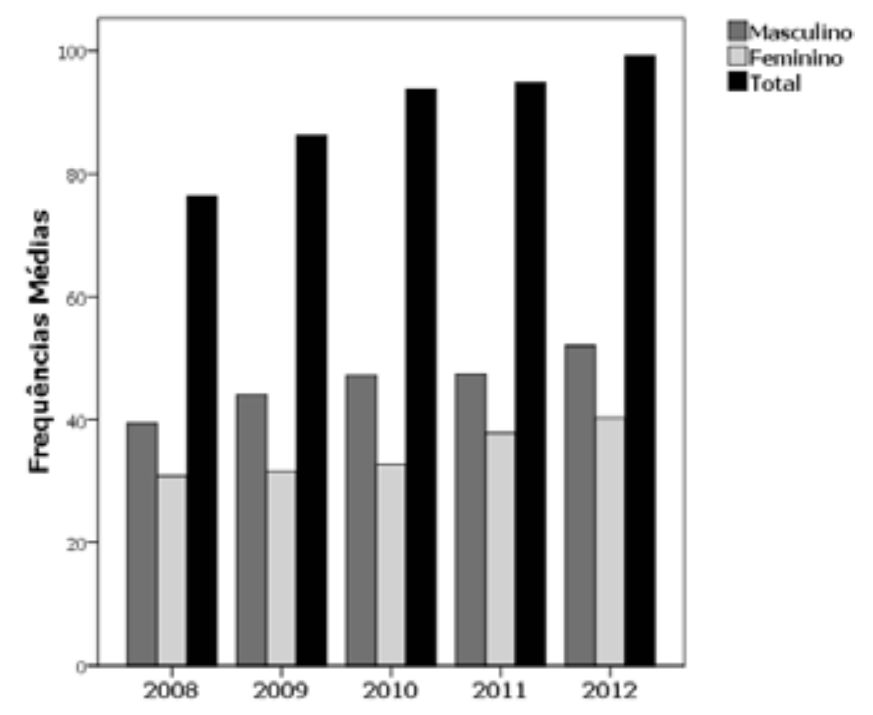

Figura 2. Números médios de casos nos sexos masculino, feminino e no total dos internamentos por câncer entre os idosos atendidos no Sistema Único de Saúde (SUS) na Paraíba entre 2008 e 2013 (mês de janeiro excluído).

As localizações mais frequentes de câncer foram as de próstata $(10,8 \%)$, mama $(9,8 \%)$, neoplasias malignas de outras localizações mal definidas, secundárias e não especificadas $(8,99 \%)$, seguidas pelo câncer de cólon $(8,05 \%)$ (Tabela 2). 
Tabela 2. Localizações de Câncer entre os Idosos Hospitalizados pelo Sistema Único de Saúde (SUS) na Paraíba no Período entre Janeiro de 2008 e Janeiro de 2013

\begin{tabular}{|c|c|c|}
\hline \multirow{2}{*}{ Neoplasias - Localização } & \multicolumn{2}{|c|}{ Frequências } \\
\hline & $f$ & $\%$ \\
\hline Próstata & 1.103 & 10,3 \\
\hline Mama & 1.008 & 9,4 \\
\hline Localizações mal definidas & 921 & 8,6 \\
\hline Cólon & 834 & 7,8 \\
\hline Estômago & 584 & 5,4 \\
\hline Esôfago & 567 & 5,3 \\
\hline Lábio, cavidade oral e faringe & 519 & 4,8 \\
\hline Tecido mesotelial e tecidos moles & 504 & 4,7 \\
\hline Reto e ânus & 434 & 4,0 \\
\hline Outras da pele & 426 & 3,9 \\
\hline Bexiga & 414 & 3,8 \\
\hline Colo do útero & 402 & 3,7 \\
\hline Pulmão & 367 & 3,4 \\
\hline Encéfalo & 282 & 2,6 \\
\hline Laringe & 253 & 2,4 \\
\hline Outras porções do útero & 247 & 2,3 \\
\hline $\begin{array}{l}\text { Doença de Hodgkin e linfoma não- } \\
\text { Hodgkin }\end{array}$ & 232 & 2,2 \\
\hline Leucemia & 226 & 2,1 \\
\hline Outros órgãos genitais femininos & 218 & 2,0 \\
\hline Pele & 207 & 1,9 \\
\hline Outros órgãos digestivos & 171 & 1,6 \\
\hline $\begin{array}{l}\text { Outros órgãos respiratórios e } \\
\text { intratorácicos }\end{array}$ & 125 & 1,2 \\
\hline Outros órgãos genitais masculinos & 113 & 1,1 \\
\hline Osso e cartilagem articular & 109 & 1,0 \\
\hline Pâncreas & 106 & 1,0 \\
\hline $\begin{array}{l}\text { Outros dos tecidos linfoides e } \\
\text { hematopoieticos }\end{array}$ & 97 & 0,9 \\
\hline Outros do trato urinário & 94 & 0,9 \\
\hline Fígado e vias biliares intra-hepáticas & 90 & 0,8 \\
\hline Outros do sistema nervoso central & 86 & 0,8 \\
\hline Olhos e anexos & 76 & 0,7 \\
\hline Total & 10.739 & 100 \\
\hline
\end{tabular}

Por faixa etária, a maior frequência observada entre 60 e 69 anos foi a categorizada como "não especificada" (559 casos), seguida pelo câncer de mama (545 casos); na faixa entre 70 e 79 anos, o câncer de próstata foi o mais frequente (462), o que foi verificado também entre os idosos com 80 anos e mais, nos quais o câncer de próstata foi o mais frequente (192 casos). Quanto às frequências percentuais por localização, o câncer de esôfago foi mais comum entre 60 e 69 anos (73,4\%), outras neoplasias geniturinárias entre 70 e 79 anos $(54,9 \%)$ e outras neoplasias de pele naqueles com 80 anos e mais (39,7\%).

$\mathrm{O}$ atendimento hospitalar foi registrado como internação em caráter de urgência em 6.895 AIH $(53,1 \%)$. As internações ocorreram em hospitais privados conveniados ao SUS em 9.397 casos (87,5\%). A maioria das internações ocorreu na cidade de João Pessoa $(7.410 / 69,4 \%)$, seguida por Campina Grande (2.910/27,1\%); apenas 418 internações foram realizadas em outras cidades da Paraíba.

A permanência hospitalar média de pacientes idosos internados por câncer foi de 5,7 dias, que não diferiu significativamente da permanência média dos idosos com todos os diagnósticos no mesmo período (5,3 dias). Ocorreram 1.448 óbitos de pacientes idosos internados com diagnóstico de câncer, o que representa $13,5 \%$ dos casos.

As frequências médias de óbitos por câncer variaram de $6,4 \%$ das médias de óbitos por todas as causas em 2009 até 10,1\% em 2012. Observou-se diferença estatisticamente significante nas frequências médias de óbitos entre os cinco anos avaliados, tanto no número de óbitos hospitalares de idosos por todas as causas $(p=0,0001)$ quanto no de óbitos por câncer $(p=0,001)$, considerando as três faixas de idade conjuntamente. As médias das taxas de mortalidade específica por todas as neoplasias foram mais elevadas nos homens (582,2/100.000 habitantes) que nas mulheres (380,1/100.000 habitantes). Os casos de câncer de próstata apresentaram as taxas absolutas de mortalidade mais elevadas entre os dados disponíveis no SIM (Tabela 3). 
Tabela 3. Taxa de Mortalidade Específica (Óbitos por 100.000 Habitantes) entre os Idosos Hospitalizados pelo Sistema Único de Saúde (SUS) na Paraíba por ano entre 2008 e 2011

\begin{tabular}{|c|c|c|c|c|}
\hline Neoplasias & 2008 & 2009 & 2010 & 2011 \\
\hline Todas as localizações & 1,94 & 1,93 & 2,04 & 2,11 \\
\hline Pulmão & 41,6 & 44,1 & 45,9 & 43,4 \\
\hline Esôfago & 21,2 & 14,9 & 14,6 & 19,4 \\
\hline Estômago & 47,1 & 40,0 & 44,8 & 42,3 \\
\hline Reto e ânus & 20,2 & 15,8 & 16,0 & 16,5 \\
\hline Mama & 41,7 & 40,0 & 39,5 & 38,8 \\
\hline Colo do útero & 20,8 & 16,9 & 15,6 & 16,7 \\
\hline Próstata & 168,0 & 145,7 & 128,9 & 139,4 \\
\hline Demais Localizações & 245,7 & 260,9 & 243,7 & 251,5 \\
\hline
\end{tabular}

Inclui todos os casos de neoplasias em idosos em cada ano considerado; Demais localizações, exceto pulmão, esôfago, estômago, reto e ânus, mama, colo do útero e próstata.

\section{DISCUSSÃO}

A frequência de hospitalizações de pacientes idosos com diagnóstico principal de câncer no sistema público de saúde do Estado da Paraíba entre 2008 e 2013 foi baixo, comparando-se os resultados encontrados com a frequência de neoplasias malignas como causa de internação entre os idosos em 2010 nos Estados do Paraná, estimada em 8,8\% (CASTRO et al., 2013), e de São Paulo, de 10\% (ALVARENGA; MENDES, 2003). Por outro lado, em estudo realizado por Martin (2006) de morbidade hospitalar da população idosa do município de Londrina (537.566 habitantes), Estado do Paraná, Brasil, no ano 2000, a partir do SIH/SUS, o grupo das neoplasias representou $11 \%$ do total, percentual inferior ao encontrado no nosso estudo. Contudo, em outro estudo também realizado através do SIH/SUS, considerando outro município (13.704 habitantes) do Estado do Paraná no ano de 2005, verificou-se que $5,1 \%$ das internações de idosos (18/352) ocorreram por diagnóstico de neoplasias (JOBIM; SOUZA; CABRERA, 2010), percentual próximo ao que se encontrou no nosso estudo.

Em um hospital universitário de Minas Gerais, Brasil, a frequência percentual de internações de idosos por câncer em relação às outras causas foi de 8,3\% (RABELO et al., 2010), também inferior ao que encontramos neste estudo. No Brasil como um todo, em 2010, as internações por neoplasias malignas em idosos foram de 4,5\% em relação a todas as causas, com taxas de $4,9 \%$ entre 60 a 69 anos e de $2,3 \%$ em idosos de 80 e mais (CHAIMOWICZ, 2013).

Em estudo realizado por Góis e Veras (2010), em que se compararam as maiores frequências de morbidade hospitalar em idosos no ano de $2005 \mathrm{com}$ as do ano de 1994 através dos dados do SIH/SUS, verificou-se que no Brasil houve o aumento em dobro das neoplasias do ano de 1994 (4\%) para 2005 (8\%), ou seja, o percentual da observação de 1994 foi semelhante ao que encontramos, mas a proporção constatada em 2005 foi quase o dobro do verificado no presente estudo. Em outro estudo com dados de idosos hospitalizados em dois hospitais universitários e dois não universitários da cidade do Rio de Janeiro no ano de 1999 (AMARAL et al., 2004), obtidos a partir do mesmo SIH/SUS utilizado no presente estudo, verificou-se que das 7.584 internações hospitalares de idosos, 794 foram motivadas por neoplasias (10,5\%), percentual também superior ao que foi encontrado nesta casuística da Paraíba. Contudo, no referido estudo, coletaram-se apenas informações referentes a hospitais de nível terciário.

A maior parte dos estudos referidos corrobora a ideia de que a representação das taxas de incidência de câncer no Brasil mostra diferença na distribuição desta doença entre as regióes do país, com maiores taxas no Sul e Sudeste, padrão intermediário na região CentroOeste, enquanto são estimadas como menores no Norte e Nordeste (DAZZI; ZATTI; BALDISSERA, 2014). Pelo 
que se observou no nosso estudo, porém, o percentual de idosos internados com neoplasias é alto quando comparado com o total de internações em todas as faixas etárias.

As internações por neoplasias foram observadas principalmente entre os homens, embora com apenas leve predominância deste sexo. A proximidade entre estes percentuais pode ser atribuída aos igualmente elevados números de casos de câncer de próstata $(10,8 \%)$ e de mama $(9,8 \%)$ observados nesta casuística. Ainda com relação ao sexo, a prevalência de câncer entre homens e mulheresé muito similar entre si nos países desenvolvidos, enquanto nos países em desenvolvimento, a prevalência nas mulheres é $25 \%$ maior, o que possivelmente reflete o predomínio, em homens, de localizações de câncer com pior sobrevida, tais como fígado, esôfago e estômago (GUERRA; GALLO; MENDONÇA, 2005). Contudo, de acordo com um estudo de Mergen e Strassburger (2009) em um Centro de Alta Complexidade que atende pacientes oncológicos de mais de 120 municípios do Estado do Rio Grande do Sul, 1.279 (51\%) dos pacientes eram do sexo feminino. Também de acordo com o Instituto Nacional de Câncer (2007), a estimativa para 2008 foi de 466.730 casos novos, dos quais o sexo feminino responderia por $50,3 \%$. Salienta-se ainda que, especificamente em relação ao sexo, existem variações distintas entre as diversas regiões do País. Análise do percentual de internações no SUS segundo tipo de causa (Capítulo da CID-10) no Estado de São Paulo em 2012 evidencia que na taxa de internação por neoplasias o sexo feminino apresenta predomínio nas faixas etárias mais jovens, mas o sexo masculino predomina a partir dos 60 anos, com taxas mais elevadas (MENDES, 2013).

A maior parte das internações de idosos foi encontrada na faixa etária de 60 a 69 anos, porém este dado não indica necessariamente que a taxa de internação neste segmento de idade foi maior do que entre os idosos de 70 a 79 anos ou com mais de 80 anos, porque o cálculo não teve por base a população total considerada, mas apenas o valor percentual de cada faixa etária para o total de registros encontrados no SIH. Este resultado foi semelhante ao reportado por outros autores (FREITAS-MATHIAS; MELO-JORGE, 2005; MARTIN et al., 2006; MENDES, 2013), que constataram ser a ocorrência de internações por neoplasias menor na faixa acima de 80 anos, mas diferiu dos resultados encontrados em um hospital público de ensino em Petrópolis, em que o maior percentual de internação $(41,8 \%)$ foi verificado entre idosos na faixa etária de 70 a 79 anos (MOTTA; HANSEL; SILVA, 2010). A menor frequência de internamentos por câncer em idosos acima de 80 anos pode estar relacionada ao fato de se tratar de uma doença com alta letalidade e de duração, em geral, relativamente curta.

As taxas de mortalidade específica foram muito mais elevadas que as estimativas para adultos não idosos. Em publicação do Instituto Nacional de Câncer que mostra análise da mortalidade em adultos de todas as idades, é demonstrado que, em 2002, o câncer de pulmão (com taxa bruta de 12,9/100.000 habitantes) foi a primeira causa de morte por câncer no sexo masculino, seguido do câncer da próstata $(9,14 / 100.000)$, do estômago (8,47/100.000), do esôfago $(4,94 / 100.000)$ e do cólon e reto $(4,10 / 100.000)$. A estimativa para o câncer da mama (10,25/100.000) em 2002 foi a primeira causa de morte por câncer entre as mulheres, seguido pelo câncer de pulmão (5,29/100.000), do cólon e reto $(4,59 / 100.000)$, do colo do útero $(4,49 / 100.000)$ e do estômago (4,24/100.000) (INSTITUTO NACIONAL DE CÂNCER, 2002). A idade é um marcador de risco importante, ganhando um significado especial no câncer de próstata, uma vez que tanto a incidência como a mortalidade aumentam exponencialmente antes dos 50 anos.

Os órgãos mais frequentemente atingidos na presente casuística corroboram a literatura no que concerne à morbidade por câncer entre os idosos, exceto pela frequência do câncer de pele, que foi de menor proporção no presente estudo. Relata-se na literatura que as frequências mais elevadas são, no homem, câncer de pele, próstata, pulmão, estômago e cólon; na mulher, câncer de mama, pele, colo do útero, cólon, estômago e pulmão, além do sistema linfático (leucemias e linfomas) em ambos os sexos (DAZZI; ZATTI; BALDISSERA, 2014). Martin et al. (2006) verificaram que as principais neoplasias detectadas em idosos em uma cidade do Sul do Brasil foram o câncer de cólon, próstata, do estômago e mama, com cerca de $1 \%$ para cada uma.

$\mathrm{O}$ câncer de próstata foi o mais frequente no sexo masculino, semelhante aos dados encontrados no 
Brasil (INSTITUTO NACIONAL DE CÂNCER, 2002). Este câncer é considerado hoje o mais prevalente em homens, representando cerca de $10 \%$ do total de neoplasias malignas, com íntima ligação ao fator idade, já que cerca de três quartos dos casos no mundo ocorrem a partir dos 60 anos. O câncer de próstata representa 9,7\% das neoplasias malignas entre os homens, 15,3\% em países desenvolvidos e 4,3\% em países em desenvolvimento (RODRIGUES; FERREIRA, 2010). No nosso estudo, o percentual encontrado foi intermediário entre estas duas taxas, corroborando o percentual geral de 9,7\%. Por outro lado, também corroborando os dados da literatura, verificou-se que o câncer de mama foi a principal neoplasia no sexo feminino, o que é reportado também no Brasil e no mundo (PARKIN; BRAY; DEVESA, 2001; INSTITUTO NACIONAL DE CÂNCER, 2006). Na atualidade, o câncer de mama é considerado o segundo tipo de câncer mais frequente no mundo, porém o mais frequente entre as mulheres. Ao contrário do câncer de colo de útero, o câncer de mama encontra-se relacionado ao processo de industrialização, com risco de adoecimento associado a elevado status socioeconômico, além de outros fatores de risco tradicionalmente descritos, tais como baixa paridade, idade precoce da menarca e tardia da menopausa, obesidade, altura e consumo de álcool (RODRIGUES; FERREIRA, 2010; MENDES, 2013). O câncer de cólon, o quarto mais frequente na presente casuística (após os casos não especificados e secundários), é, mundialmente, a quarta neoplasia mais incidente em ambos os sexos (INSTITUTO NACIONAL DE CÂNCER, 2006).

As neoplasias de mama, próstata e cólon atingem principalmente pessoas de condições socioeconômicas mais altas (ROSAS et al., 2013). Assim, os dados encontrados no presente estudo sugerem uma transição epidemiológica em andamento. Como se observou, ao mesmo tempo em que é nítida a maior prevalência de neoplasias associadas a um melhor nível socioeconômico - mama, próstata e cólon-simultaneamente, observaramse frequências também elevadas de tumores geralmente associados à pobreza - colo do útero, estômago e cavidade oral (INSTITUTO NACIONAL DE CÂNCER, 2006). Esta distribuição talvez resulte de exposições diferenciadas a fatores ambientais relacionados ao processo de industrialização, como agentes químicos, físicos e biológicos, e das condições de vida, que variam de intensidade em função das desigualdades sociais. Assim, verifica-se a existência concomitante de tumores típicos das regióes pouco desenvolvidas com aqueles de alta incidência em países desenvolvidos, fruto da coexistência de fatores de risco tradicionais e modernos, aos quais a população brasileira se encontra exposta (ROSAS et al., 2013). Contudo, é preciso levar em conta que, ao se considerar que a distribuição dos casos de câncer segundo o tipo de tumor nas cinco regiões do país, observa-se elevada heterogeneidade, o que também se verifica entre os diferentes Estados da Federação, possivelmente pelas grandes dimensões territoriais do Brasil que, por sua vez, associam-se a marcadas diferenças regionais em aspectos culturais, sociais e econômicos (FACINA, 2011).

A distribuição dos diagnósticos mais frequentes em idosos demonstra que entre as três primeiras causas de óbito, as neoplasias são a segunda maior causa, depois das doenças cardiovasculares (FREITAS-MATHIAS; MELOJORGE, 2005). Neste último estudo referido, na análise da mortalidade proporcional por neoplasias em Maringá (PR), verificada a partir de dados do Instituto Brasileiro de Geografia e Estatística, tanto de idosos hospitalizados como não hospitalizados, as taxas variaram de 12,5\% (1979-1981) a 17,9\% (1996-1998), superiores, portanto, aos encontrados neste estudo considerando idosos que morreram nas internações por neoplasias em relação ao total de idosos internados na Paraíba no período de observação. É necessário ponderar novamente que a mortalidade por neoplasias, assim como a sua própria prevalência, apresenta grande variação entre as unidades da Federação (INSTITUTO NACIONAL DE CÂNCER, 2006).

De acordo com estudo de Amaral et al. (2004), a mortalidade de pacientes idosos hospitalizados por câncer em quatro grandes hospitais do Rio de Janeiro foi de $16,9 \%$, percentual superior ao do presente estudo $(10,2 \%)$. A razão de coeficiente de mortalidade deste foi calculada para se observar o comportamento ou a importância que cada diagnóstico representou como causa de internação em relação à causa de óbito. Devese salientar novamente que, como evento, a doença não 
é única, e os dados de internação não são referentes ao indivíduo, e sim ao montante das internações realizadas no período. Quando se consideram as cinco primeiras localizações primárias dos tumores que mais levaram ao óbito no Brasil (para os homens e mulheres, respectivamente: pulmão, estômago, próstata, esôfago e medula óssea (leucemias) e mama, colo uterino, pulmão, estômago e intestino grosso) (FACINA, 2011), verifica-se que entre estas estão mama, próstata e cólon, as principais localizações verificadas entre os idosos paraibanos neste estudo.

No perfil de internações hospitalares em idosos no município de Maringá no ano de 1998, as neoplasias constituíram o motivo de internação que apresentou maior diferença se comparadas ao padrão de distribuição das causas de óbito, com coeficiente de mortalidade de 0,7 , muito abaixo das encontradas nas três faixas etárias consideradas no presente estudo (MOTTA; HANSEL; SILVA, 2010). Os autores do referido estudo consideraram que o resultado encontrado deveuse provavelmente à maior frequência de internações por câncer para intervenções cirúrgicas, mas a prevalência de internações por neoplasias nesse caso foi de $2,8 \%$. A razão de coeficiente de mortalidade deve ser influenciada por vários fatores mas, basicamente, relaciona-se à gravidade de cada tipo de neoplasia.

A duração das internações, neste estudo, pode ser considerada compatível com o que é observado na clientela idosa, independente da categoria de diagnóstico que motivou a internação (ALVARENGA; MENDES, 2003; MOTTA; HANSEL; SILVA, 2010), assim como com a média de permanência hospitalar no SUS, considerando todas as idades e diagnósticos, que foi de 5,8 dias em 2007 (RABELO et al., 2010). Os dados encontrados no presente estudo revelaram similitude com os resultados apresentados sobre análise das informações obtidas na base de dados do SIH/SUS no Estado do Rio Grande do Sul, e incluídas no sistema entre os meses de janeiro a novembro do ano de 2013, que revelou predomínio de neoplasias na faixa etária de 60 a 69 anos (DAZZI; ZATTI; BALDISSERA, 2014).

Quanto ao caráter de atendimento, os dados observados no presente estudo foram semelhantes aos encontrados por Dazzi, Zatti e Baldissera (2014) no
Estado do Rio Grande do Sul, em que as internações foram em caráter de urgência em 58,1\% das AIH. Porém, nosso estudo diferiu dos referidos autores quanto à proporção dos atendimentos de pacientes oncológicos em instituições privadas, pois no Rio Grande do Sul em 2013 , foi de $65,5 \%$, percentual inferior ao encontrado no nosso estudo.

Entre as limitações do presente estudo, salienta-se que os resultados encontrados devem ser interpretados levando-se em conta que os bancos de dados disponíveis no Datasus não estão livres de falhas na sua tabulação. Contudo, os estudos epidemiológicos de base populacional mais adequados para a produção de informações acerca das condições de saúde apresenta custo e complexidade operacional que muitas vezes impossibilitam sua utilização. Os dados secundários gerados pelos sistemas nacionais de informação em saúde são uma boa alternativa neste caso. Por outro lado, não se usaram taxas de internação na análise dos dados deste estudo porque os valores totais da população incluiriam também o contingente de pessoas atendidas no sistema privado de saúde do Estado, que não são cobertas pelo SIH-SUS.

O aumento crescente do número de diagnósticos de câncer entre os anos avaliados neste estudo, embora não estatisticamente significativo, pode se relacionar à melhoria das informações observada na última década no País em relação aos sistemas de registros coletados sistematicamente pelo Ministério da Saúde (SILVA; NORONHA; SANTOS, 2008; RIBEIRO, 2009).

É preciso considerar que existem limitações nas estatísticas produzidas pelos registros hospitalares. Estes são considerados seletivos por se tratarem de dados relativos às doenças graves, e também parciais, por ser conhecido apenas parte desse contingente (SUS). No entanto, como o setor público é o maior financiador das hospitalizações, sendo responsável pela assistência médico-hospitalar a aproximadamente $80 \%$ da população brasileira, sua análise significa conhecer a grande maioria das internações no Brasil (SISTEMA NACIONAL DE VIGILÂNCIA EM SAÚDE, 2011).

Por outro lado, informações hospitalares do SUS referem-se às internações e não aos indivíduos, podendo existir, desta forma, uma superestimação da prevalência 
de certas doenças que requerem internações sucessivas, como é o caso das neoplasias em pacientes idosos. Assim, é possível que um mesmo paciente tenha sido internado mais de uma vez no período de observação (RIBEIRO, 2009). Desta forma, o número de internações pode ter excedido o número de pessoas internadas nos anos considerados, e deve-se ter em mente que a prevalência encontrada não reflete necessariamente a prevalência de neoplasias em idosos da população.

Observações também devem ser feitas no que concerne à qualidade das informações contidas nas AIH. $\mathrm{O}$ diagnóstico de internação pode ser uma variável de menor confiabilidade, em virtude da finalidade contábil da AIH, pois os hospitais podem ter selecionado diagnósticos que permitissem maior remuneração. Contudo, trabalhos na área de gerontologia que tiveram como fonte a utilização dos dados do Datasus apresentaram elevado nível de aceitação pela comunidade científica, sendo seus resultados revertidos em subsídios para o próprio SUS (ALVARENGA; MENDES, 2003; CASTRO et al., 2013). Análise de confiabilidade de diagnósticos das AIH desenvolvida no Rio de Janeiro mostrou que, em geral, os resultados naquele município foram melhores do que se esperava para o banco de dados SIH/SUS (SISTEMA NACIONAL DE VIGILÂNCIA EM SÁUDE, 2011). Análise semelhante foi realizada para as internações ocorridas entre 1995 e 1998 no município de Maringá (PR), Brasil, concluindo-se que melhor confiabilidade foi encontrada para os diagnósticos mais frequentes e quando estes foram analisados segundo agrupamentos e capítulos da CID-10 (FREITAS-MATHIAS; MELO-JORGE, 2005).

Em geral, no Brasil, análises da situação de saúde dos idosos ficam comprometidas pela elevada proporção de registros de causas mal definidas nesta faixa etária, afetando, assim, estudos de morbidade (VERAS; MARTINS, 1994; INSTITUTO NACIONAL DE CÂNCER, 2003; MELLO-JORGE; LAURENTI; GOTLIEB, 2009; MENDES, 2010; ABREU; SAKURAI; CAMPOS, 2010). Entretanto, o percentual de $10 \%$, considerado como inadequado para fins de processamento estatístico (OLIVEIRA-CAMPOS; CERQUEIRA; RODRIGUES NETO, 2011), não foi alcançado no presente estudo.

Assim, a despeito das limitações referidas, os resultados encontrados representam indícios de que a prevalência de internações por câncer em idosos pelo SUS na Paraíba é inferior aos de outros Estados do Brasil, porém a gravidade do problema ainda é evidente, já que o câncer atinge a clientela acima de 60 anos em $25 \%$ dos hospitalizados no SUS pela doença no Estado, e que sua mortalidade é elevada.

\section{CONCLUSÕES}

Os resultados encontrados neste estudo indicam uma prevalência de internações hospitalares na população idosa na Paraíba por câncer inferior aos de outros Estados do Brasil, mas representando percentual importante em relação ao número total de hospitalizações por câncer no Estado, com maiores frequências de câncer de próstata, mama e cólon, além de apontar para sua elevada mortalidade hospitalar. Esses dados reforçam a necessidade de estratégias de promoção de saúde e controle adequado de neoplasias na população geriátrica, contribuindo para a reflexão de ações promovidas no setor saúde pela gestão pública do Estado do Paraíba e outros Estados brasileiros.

\section{REFERÊNCIAS}

ABREU, D. M. X.; SAKURAI, E.; CAMPOS, L. N. A evolução da mortalidade por causas mal definidas na população idosa em quatro capitais brasileiras, 1996-2007. Rev Bras Estud Popul., São Paulo, v. 27, n. 1, p. 75-88, jun. 2010.

ALVARENGA, M. R. M.; MENDES, M. M. R. O perfil das readmissões de idosos num hospital geral de Marília/SP. Rev Latinoam Enferm, Ribeirão Preto, v. 1, n. 3, p. 305311, jun. 2003.

AMARAL, A. C. S. et al. Perfil de morbidade e de mortalidade de pacientes idosos hospitalizados. Cad Saúde Pública, Rio de Janeiro, v. 20, n. 6, p. 1617-1626, dez. 2004.

AMERICAN CANCER SOCIETY. Cancer Facts and Figures 2005. Disponível em: < http://www.cancer.org/ docroot/STT/stt_0.asp > . Acesso em: 05 ago. 2015.

ASSIS, C. M. R. B. et al. Oncologia geriátrica: conceitos, tendências e desafios. Geriatria \& Gerontologia, Fortaleza, v. 5, n. 2, p. 106-111, abr/jun. 2011. 
BRASIL. INSTITUTO BRASILEIRO DE GEOGRAFIA E ESTATÍSTICA. Censo Demográfico, 2010: Base de dados na Internet. Brasília: IBGE; 2010. Disponível em: $\quad<$ http://www.ibge.gov.br/estadosat/temas. php? sigla $=$ pb\&tema $=$ censodemog2010_amostra $>$. Acesso em: 05 ago. 2015.

BRASIL. Ministério da Saúde. Instituto Nacional de Câncer. Estimativas da incidência e mortalidade por câncer. Rio de Janeiro: INCA, 2003.

BRASIL. Ministério da Saúde. Secretaria de Assistência à Saúde. Instituto Nacional de Câncer. Programa nacional de controle do câncer da próstata: documento de consenso. Rio de Janeiro: INCA, 2002.

BRASIL. Ministério da Saúde. Secretaria de Atenção à Saúde. Instituto Nacional de Câncer. Coordenação de Prevenção e Vigilância de Câncer. Estimativas 2008: Incidência de câncer no Brasil. Rio de Janeiro: Inca, 2007. Disponível em: <http://www.inca.gov.br/ estimativa/2008>. Acesso em: 20 set. 2015.

BRASIL. Ministério da Saúde. Secretaria de Atenção à Saúde. Instituto Nacional de Câncer. A situação do câncer no Brasil. Rio de Janeiro: INCA, 2006.

BRASIL. Sistema Nacional de Vigilância em Saúde. Brasília: Ministério da Saúde, 2011.

CASTRO, V. C. et al. Perfil de internações hospitalares de idosos no âmbito do sistema único de saúde. Rev Rene, Fortaleza, v. 14, n. 4, p. 791-800, 2013.

CHAIMOWICZ, F. Saúde do idoso. 2. ed. Belo Horizonte: NESCON UFMG, 2013.

DAZZI, M. C.; ZATTI, C. A.; BALDISSERA, R. Internações Hospitalares por Neoplasias no Estado do Rio Grande do Sul. Braz J Surg Clin Res., v. 7, n. 2, p. 05-09, jun/ago. 2014.

FACINA, T. Estimativa 2012: Incidência de Câncer no Brasil. Rev Bras Cancerol., Rio de Janeiro, v. 57, n. 4, p. 557, 2011.

FREITAS-MATHIAS, T. A.; MELO-JORGE, M. H. P. Hospitalização e mortalidade em idosos: um exercício de análise comparativa. Ciênc Cuid Saúde, Maringá, v. 4, n. 1, p. 25-36, jan/abr. 2005.
GÓIS, A. L. P.; VERAS, R. P. Informações sobre a mortalidade hospitalar em idosos nas internações do Sistema Único de Saúde do Brasil. Ciênc saúde coletiva, Rio de Janeiro, v. 15, n. 6, p. 2859-2869, set. 2010.

GUERRA, M. R.; GALLO, C. M.; MENDONÇA, G. A. S. Risco de câncer no Brasil: tendências e estudos epidemiológicos mais recentes. Rev Bras Cancerol., Rio de Janeiro, v. 51, n. 3, p. 227-234, 2005.

JOBIM, E. F. C.; SOUZA, V.; CABRERA, M. A. S. Causas de hospitalização de idosos em dois hospitais gerais pelo Sistema Único de Saúde (SUS). Acta Scient Health Sci., Maringá, v. 32, n. 1, p. 79-83, 2010.

LIMA-E-COSTA, M. F. F. et al. Diagnóstico da situação de saúde da população idosa brasileira: um estudo da mortalidade e das internações hospitalares públicas. Inf Epidemiol Sus, Brasília, v. 9, n. 1, p. 43-50, jan/mar. 2000 .

LOYOLA FILHO, A. I. et al. Causas de internações hospitalares entre idosos brasileiros no âmbito do Sistema Único de Saúde. Epidemiol. Serv. Saúde, Brasília, v. 13, n. 4, p. 229-238, dez. 2004.

MARTIN, G. B. et al. Assistência hospitalar à população idosa em cidade do sul do Brasil. Epidemiol Serv Saúde, Brasília, v. 15, n. 1, p. 59-65, jan/mar. 2006.

MELLO-JORGE, M. H. P.; LAURENTI, R.; GOTLIEB, S. L. D. O. Sistema de informações sobre Mortalidade - SIM: Concepção, implantação e avaliação. In: MINISTÉRIO DA SAÚDE. Organização Pan-Americana da Saúde, Fundação Oswaldo Cruz. A experiência brasileira em sistemas de informação em saúde. Brasília: Ministério da Saúde, 2009.

MENDES, J. D. V. M. Morbidade nas internações de idosos no SUS/SP em 2010-2011. Boletim Eletrônico do Grupo Técnico de Avaliação e Informações de Saúde, São Paulo, v. 3, n. 9, p. 1-20, 2010.

MENDES, P. D. V. Morbidade das Internações em 2012 no SUS do Estado de São Paulo. Boletim Eletrônico do Grupo Técnico de Avaliação e Informações de Saúde, São Paulo, v. 5, n. 19, p. 1-12, 2013. 
MERGEN, C. T. T.; STRASSBURGER, S. Z. Prevalência dos Tipos de Câncer em Pacientes Atendidos em um Centro de Alta Complexidade em Oncologia. Rev Contexto \& Saúde, Ijuí, v. 9, n. 17, p. 31-38, jul./dez. 2009.

MORAES, M. F. Câncer e Velhice no Brasil. Rev Bras Cancerol., Rio de Janeiro, v. 43, n. 1, jan./mar. 1997.

MOTTA, C. C. R.; HANSEL, C. G.; SILVA, J. Perfil de internações de pessoas idosas em um hospital público. Rev Eletr Enf, Goiânia, v. 12, n. 3, p. 471-477, set. 2010.

OLIVEIRA-CAMPOS, M.; CERQUEIRA, M. B. R.; RODRIGUES NETO, J. F. Dinâmica populacional e o perfil de mortalidade no município de Montes Claros (MG). Ciênc. saúde coletiva, Rio de Janeiro, v. 16, suppl.1, p. 1303-1310, 2011.

ORGANIZAÇÃO MUNDIAL DA SAÚDE. Classificação Estatística Internacional de Doenças e Problemas Relacionados à Saúde. 2. ed. São Paulo: Edusp, 1995.

PARKIN, D. M.; BRAY, F. I.; DEVESA, S. S. Cancer burden in the year 2000. The global picture. Eur J Cancer, v. 37, suppl. 8, n. 6, p. 4-66, jul. 2001.

RABELO, L. P. O. et al. Perfil de pacientes idosos internados em um hospital universitário. Rev Min Enferm., Belo Horizonte, v. 14, n. 3, p. 293-300, jul./set. 2010.

RIBEIRO, J. M. Desenvolvimento do SUS e racionamento de serviços hospitalares. Ciênc. saúde coletiva, Rio de Janeiro, v. 14, n. 3, p. 771-782, maio/jun. 2009.

RODRIGUES, J. S. M.; FERREIRA, N. M. L. A. Caracterização do Perfil Epidemiológico do Câncer em uma Cidade do Interior Paulista: Conhecer para Intervir. Rev Bras Cancerol., Rio de Janeiro, v. 56, n. 4, p. 431-441, out./ dez. 2010.

ROSAS, M. S. L. et al. Incidência do Câncer no Brasil e o Potencial Uso dos Derivados de Isatinas na Cancerologia Experimental. Rev Virtual Quim., São Paulo, v. 5, n. 2, p. 243-265, mar/abr. 2013.

SILVA, G. A.; NORONHA, C. P.; SANTOS, M. O. et al. Diferenças de gênero na tendência de mortalidade por câncer de pulmão nas macrorregiões brasileiras.Rev Bras Epidemiol., São Paulo, v. 11, n. 3, p. 411-419, set. 2008.
SIQUEIRA, A. B. et al. Impacto funcional da internação hospitalar de pacientes idosos.Rev Saúde Pública, São Paulo, v. 38, n. 5, p. 687-694, out. 2004.

VALLGARDA, S. Is old age necessarily connected with high hospital admission rates? Scand J Public Health, v. 2, n. 2, p. 137-142, jun. 1999.

VERAS, C. M. T.; MARTINS, M. S. A confiabilidade dos dados nos formulários de Autorização de Internação Hospitalar (AIH), Rio de Janeiro, Brasil. Cad Saúde Pública, Rio de Janeiro, v. 10, n. 3, p. 339-355, jul./ set.1994.

Recebido em: 22 de setembro de 2015 Aceito em: 08 de novembro de 2015 